\title{
Temperature stabilization of optofluidic photonic crystal cavities
}

Kamutsch, Christian; Smith, Cameron L.C.; Graham, Alexandra; Tomljenovic-Hanic, Snjezana; McPhedran, Rose; Eggleton, Benjamin J.; O'Faolain, Liam; Krauss, Thomas F.; Xiao, Sanshui; Mortensen, Asger

Published in:

Applied Physics Letters

Link to article, DOI:

$10.1063 / 1.3152998$

Publication date:

2009

Document Version

Publisher's PDF, also known as Version of record

Link back to DTU Orbit

Citation (APA):

Kamutsch, C., Smith, C. L. C., Graham, A., Tomljenovic-Hanic, S., McPhedran, R., Eggleton, B. J., O'Faolain, L., Krauss, T. F., Xiao, S., \& Mortensen, A. (2009). Temperature stabilization of optofluidic photonic crystal cavities. Applied Physics Letters, 94(23), 231114. https://doi.org/10.1063/1.3152998

\section{General rights}

Copyright and moral rights for the publications made accessible in the public portal are retained by the authors and/or other copyright owners and it is a condition of accessing publications that users recognise and abide by the legal requirements associated with these rights.

- Users may download and print one copy of any publication from the public portal for the purpose of private study or research.

- You may not further distribute the material or use it for any profit-making activity or commercial gain

- You may freely distribute the URL identifying the publication in the public portal 


\title{
Temperature stabilization of optofluidic photonic crystal cavities
}

\author{
Christian Karnutsch, ${ }^{1, a)}$ Cameron L. C. Smith, ${ }^{1}$ Alexandra Graham, ${ }^{1}$ \\ Snjezana Tomljenovic-Hanic, ${ }^{1}$ Ross McPhedran, ${ }^{1}$ Benjamin J. Eggleton, ${ }^{1, b)}$ \\ Liam O'Faolain, ${ }^{2}$ Thomas F. Krauss, ${ }^{2}$ Sanshui Xiao, ${ }^{3}$ and N. Asger Mortensen ${ }^{3}$ \\ ${ }^{1}$ Institute of Photonics and Optical Science (IPOS), Centre for Ultrahigh-bandwidth Devices \\ for Optical Systems (CUDOS), University of Sydney, School of Physics, New South Wales 2006, Australia \\ ${ }^{2}$ School of Physics and Astronomy, University of St. Andrews, St. Andrews, Fife KY16 9SS, Scotland \\ ${ }^{3}$ Department of Photonics Engineering, Technical University of Denmark, DTU Fotonik, \\ 2800 Kongens Lyngby, Denmark
}

(Received 3 March 2009; accepted 20 May 2009; published online 9 June 2009)

\begin{abstract}
We present a principle for the temperature stabilization of photonic crystal $(\mathrm{PhC})$ cavities based on optofluidics. We introduce an analytic method enabling a specific mode of a cavity to be made wavelength insensitive to changes in ambient temperature. Using this analysis, we experimentally demonstrate a PhC cavity with a quality factor of $Q \approx 15000$ that exhibits a temperature-independent resonance. Temperature-stable cavities constitute a major building block in the development of a large suite of applications from high-sensitivity sensor systems for chemical and biomedical applications to microlasers, optical filters, and switches. () 2009 American Institute of Physics. [DOI: 10.1063/1.3152998]
\end{abstract}

Photonic crystal $(\mathrm{PhC})$ cavities have received enormous interest in recent years due to their ability to confine light on the wavelength scale. ${ }^{1} \mathrm{PhC}$ cavities may exhibit high quality factors, ${ }^{2}$ suggesting great potential for realizing compact and high-resolution refractive index sensors, ${ }^{3,4}$ including sensor arrays for biomolecular detection. ${ }^{5}$ In this context, the combination of $\mathrm{PhC}$ components with microfluidics-a subset of optofluidics ${ }^{6}$ - has attracted much attention because it allows hybrid architectures that offer tunable $e^{7,8}$ or reconfigurable ${ }^{9}$ optical properties and double-heterostructure $\mathrm{PhC}$ cavities with high quality factors of the order of $Q \approx 60000 .^{10} \mathrm{In}$ many sensor applications, a major challenge is the susceptibility of the sensing system to environmental changes. Various approaches have been reported in the literature to reduce or eliminate the detrimental cross sensitivity to ambient temperature, including Mach-Zehnder ${ }^{11,12}$ and Fabry-Pérot ${ }^{13}$ interferometric structures, structurally modifying the PhC substrate material, ${ }^{14}$ or filling the holes of a microstructured optical fiber with a suitable material. ${ }^{15}$

In this letter, we present a theoretical and experimental investigation of the temperature dependence for optofluidic cavities in planar $\mathrm{PhC}$ structures. We introduce an analytic method for designing these cavities for a specific mode to be wavelength-insensitive to changes in ambient temperature. Using this analysis, we experimentally demonstrate a temperature-independent resonance for an optofluidic $\mathrm{PhC}$ cavity. Removing the temperature dependence on the resonant wavelength promotes the development of environmentally independent high-sensitivity sensor systems and precision nanophotonic components, such as microlasers, optical filters, and switches.

In general, $\mathrm{PhC}$ cavities are created by introducing a local defect in the periodic lattice of holes, ${ }^{8}$ which requires nanometer-scale fabrication accuracy, ${ }^{16}$ whereas in this

\footnotetext{
${ }^{a)}$ Electronic mail: c.karnutsch@ physics.usyd.edu.au.

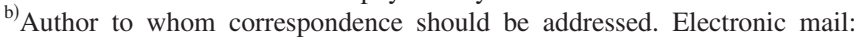
egg@physics.usyd.edu.au. Tel.: +61-2-9351-3604. FAX: +61-2-93517726.
}

work, the cavity is formed by the introduction of a fluid into air holes of the PhC. The key principle behind our optofluidic temperature stabilization is the concept that a substance (in the present study a liquid) with negative thermo-optic coefficient balances the thermal drift of the host $\mathrm{PhC}$ material. Figure 1 illustrates the refractive index temperature dependence for silicon and an infiltration liquid (e.g., Cargille immersion oil type B). For a $\mathrm{PhC}$ waveguide whose air holes have been infiltrated with another material, the effective refractive index experienced by a particular guided mode depends on the combination of the two refractive indices $(\mathrm{PhC}$ substrate material and infiltrated material), weighted by the filling fraction $f_{i}=\int_{i} n_{i}^{2}|E(r)|^{2} d r / \int n_{i}^{2}|E(r)|^{2} d r$, which gives

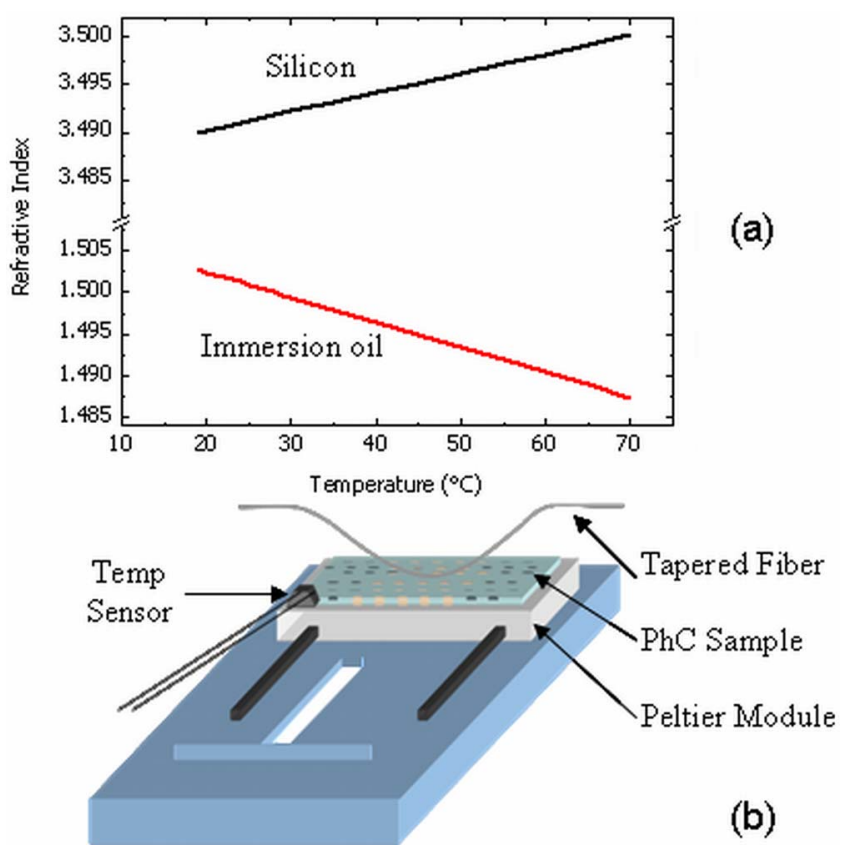

FIG. 1. (Color online) (a) Refractive index variation with temperature for silicon and Cargille immersion oil type $B$ at $\lambda \approx 1410 \mathrm{~nm}$. (b) Experimental setup. 
the relative electric field overlap with the corresponding material. Given that the thermo-optic coefficient for most common PhC substrate materials ( $\mathrm{Si}, \mathrm{III}-\mathrm{V}$ semiconductors, etc.) is positive, ${ }^{17}$ while it is negative for most liquids ${ }^{18}$ and polymers, ${ }^{19}$ there is a large range of material combinations for which the effective index of the combination may be rendered temperature insensitive if the guided mode has an appropriate portion of electric field overlap in each material.

We now express this concept mathematically by considering the field distribution in a resonant fluid-infiltrated $\mathrm{PhC}$ cavity. To calculate the change in resonance frequency with temperature, we use standard electromagnetic perturbation theory, ${ }^{20}$ linearizing the refractive index around a reference temperature $T_{0}$. For a two-component structure, such as a silicon membrane $\mathrm{PhC}$ with an infiltrated liquid, we find for the change of resonant wavelength, $\Delta \lambda$

$$
\frac{\Delta \lambda}{\Delta T} \cong \lambda\left(T_{0}\right)\left[\frac{1}{n_{1}\left(T_{0}\right)} \frac{\partial n_{1}}{\partial T} f_{1}+\frac{1}{n_{2}\left(T_{0}\right)} \frac{\partial n_{2}}{\partial T}\left(1-f_{1}\right)\right],
$$

where $\lambda$ is the free-space wavelength of the resonance and $n_{i}$ and $\partial n_{i} / \partial T$ is the refractive index and thermo-optic coefficient of material $i$ (silicon, infiltrated liquid), respectively. In Eq. (1) we have implicitly assumed that $f_{1}+f_{2}=1$, i.e., the mode only occupies the two materials, corresponding to perfect wetting conditions and hence complete hole filling and coverage of the porous membrane structure. This is only strictly true in two dimensions, not in three dimensions, but the agreement between experimental and theoretical results (see below) using Eqs. (1) and (2) shows that our assumptions are indeed justified. From Eq. (1) we can calculate the wavelength $\lambda_{\text {Stab }}$, which separates resonances with a positive and a negative temperature-induced wavelength shift. The filling fraction $f_{L}$ at which this occurs is determined by

$$
f_{L}\left(\lambda_{\mathrm{Stab}}\right)=\left[1-\frac{n_{s i}}{n_{L}} \frac{\frac{\partial n_{L}}{\partial T}}{\frac{\partial n_{S i}}{\partial T}}\right]^{-1}
$$

For a given $\mathrm{PhC}$ cavity design, $\lambda_{\text {Stab }}$ can be derived from a numerical calculation of the filling fraction. ${ }^{20} \mathrm{At}$ the wavelength $\lambda_{\text {Stab }}$ the mode profile is such that the negative thermo-optic coefficient of the infiltrated liquid $\left(\partial n_{L} / \partial T=-3 \times 10^{-4} \mathrm{~K}^{-1}\right.$ for Cargille immersion oil type B) compensates the intrinsic positive thermo-optic coefficient of the $\mathrm{PhC}\left(\partial n_{\mathrm{Si}} / \partial T=+2 \times 10^{-4} \mathrm{~K}^{-1}\right.$ for silicon $)$. From Eq. (2) it follows that temperature stabilization requires $f_{L}\left(\lambda_{\text {Stab }}\right)$ $\sim 22 \%$ for $n_{L}=1.5015, n_{\mathrm{Si}}=3.49$ and the above-mentioned thermo-optic coefficients. To experimentally achieve temperature stability for a given $\mathrm{PhC}$ structure, the interplay between the geometry of the cavity and the thermo-optic coefficient $\partial n_{L} / \partial T$ of the infiltrated liquid must be optimized. In other words, the cavity design should support a resonance at the wavelength $\lambda_{\text {Stab }}$ and at the same time that mode must satisfy $f_{L}\left(\lambda_{\text {Stab }}\right) \sim 22 \%$ (for a $\mathrm{Si} \mathrm{PhC}$ ) so that the two temperature-based effects balance. Our optofluidic scheme can be used to align a Fabry-Pérot resonance with $\lambda_{\text {Stab }}$ by changing the cavity length with an increase or decrease in size of the infiltrated region. Therefore we can tune the resonant frequencies and the filling fractions independently, which cannot be achieved with standard cavity designs that rely on photolithographic methods.

In our experiments, we use a tapered glass microtip with apex diameter $\varnothing=220 \mathrm{~nm}$ to infiltrate the optofluidic cavi-

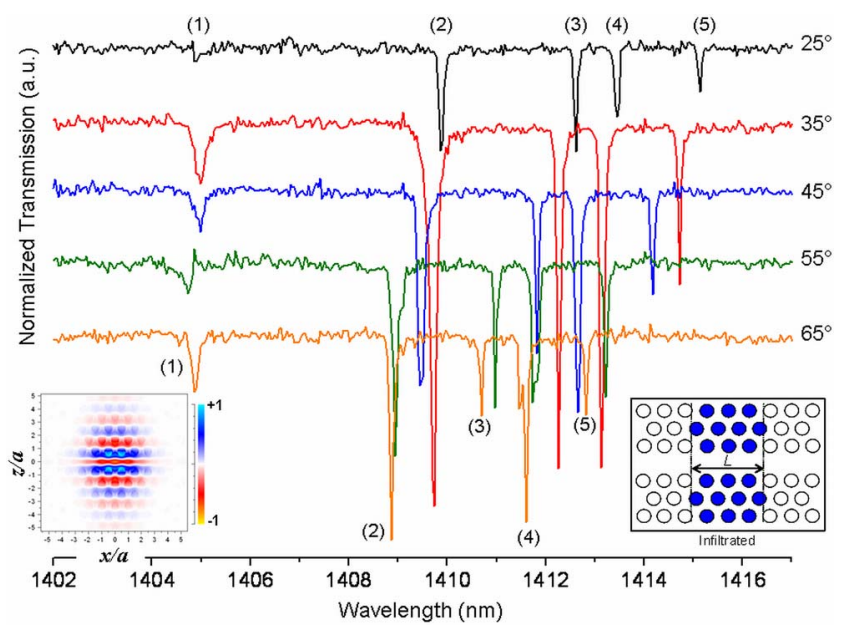

FIG. 2. (Color online) Normalized transmission spectra as a function of temperature while probing an optofluidic $\mathrm{PhC}$ cavity of length $L \approx 6.8 \mu \mathrm{m}$. Resonance peaks are labeled for clarification. Insets: (Left) Calculated major electric field component in the plane, $E_{x}$, for resonance (1). (Right) Schematic of an optofluidic PhC cavity.

ties into silicon PhC structures. The microtip is controlled by a nanopositioning stage and is inserted within a meniscus of the filling liquid (Cargille immersion oil type $\mathrm{B}, n_{L}=1.5015$ at $\lambda=1415 \mathrm{~nm}, T=292 \mathrm{~K}){ }^{21}$ As the tip is withdrawn, droplets form along its length, which are then deposited on the substrate beside the PhC structure. Finally, the microtip is used to draw a chosen droplet of appropriate volume along the $\mathrm{PhC}$, perpendicular to the waveguide, to create a doubleheterostructure type cavity. ${ }^{22}$ We have infiltrated microfluidic cavities into W0.9 PhC silicon edge-supported membranes with a slab thickness of $220 \mathrm{~nm}\left(n_{\mathrm{Si}}=3.49\right.$ at $\lambda=1407 \mathrm{~nm}$, $T=292 \mathrm{~K}) .^{23}$ The triangular PhC lattice has a periodicity of $a=410 \mathrm{~nm}$ and the hole diameter is $d=265 \mathrm{~nm}$.

We investigate the temperature-dependence of the optical properties of the cavities via an evanescent coupling technique. ${ }^{24}$ We bring a tapered fiber (diameter $\approx 1.3 \mu \mathrm{m}$ ) into close proximity above the $\mathrm{PhC}$ cavity, carefully ensuring not to touch the sample. The evanescent field significantly extends beyond the tapered fiber boundary, allowing it to interact with the optofluidic cavity. The coupling efficiency is maximum for a wavevector where the dispersion curves of the tapered fiber and waveguide mode intersect; however, by using a highly curved taper, coupling can occur for a range of wavevectors surrounding the maximum coupling point. ${ }^{24}$

The infiltrated $\mathrm{PhC}$ sample is mounted on a Peltier module to vary the temperature from 25 to $65^{\circ} \mathrm{C}$ (see Fig. 1). Figure 2 shows transmission spectra as a function of temperature while probing an optofluidic cavity of length $L$ $\approx 6.8 \mu \mathrm{m}$. We observe Fabry-Pérot resonances sustained by the microfluidic cavity that exhibit moderate quality factors, e.g., $Q \approx 15000$ for resonance (1) and $Q \approx 20000$ for resonance $(2)$. We have shown previously that the quality factor associated with the modes of optofluidic cavities decreases with decreasing wavelength. ${ }^{9}$ This phenomenon is based on the fact that the modes at shorter wavelengths experience a lower effective refractive index and thus the vertical confinement in the slab is less, reducing the quality factor of that specific mode. The depth of the Fabry-Pérot dip for the various modes varies with temperature due to the change of coupling efficiency between tapered fiber and optofluidic cavity with temperature. We note that this is a consequence of our 


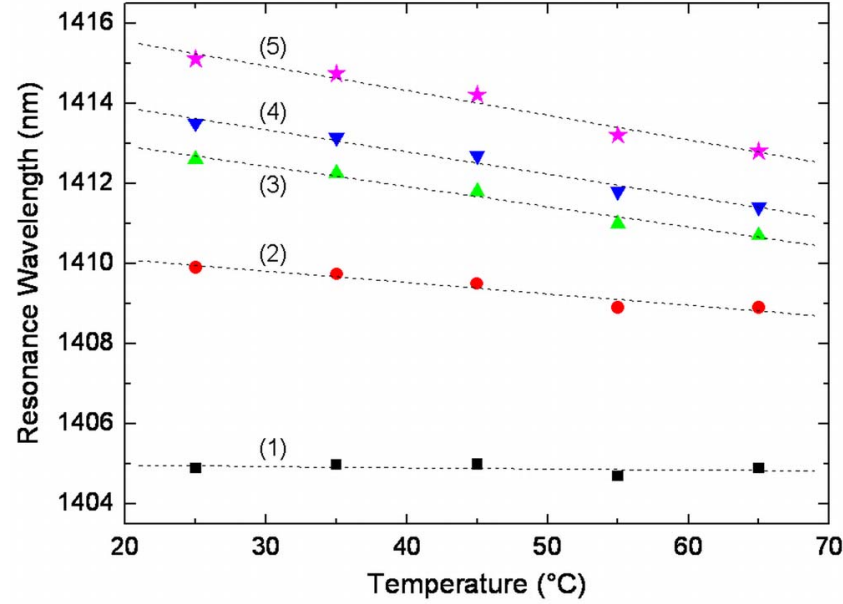

FIG. 3. (Color online) Wavelength shift as a function of temperature for the Fabry-Pérot resonances labeled in Fig. 2.

characterization method and is not an inherent property of the investigated optofluidic cavities. The resonance wavelengths shift with respect to temperature with different gradients. Figure 3 displays the wavelength shift as a function of temperature for the Fabry-Pérot resonances labeled in Fig. 2. In the investigated temperature range, resonances (2)-(5) show a blueshift of resonant wavelength between $-0.03 \mathrm{~nm} \mathrm{~K}^{-1}$ (2) and $-0.06 \mathrm{~nm} \mathrm{~K}^{-1}$ (5), while resonance (1) remains exceptionally stable at $\lambda=1405 \mathrm{~nm}$, with an extremely low gradient of $0.003 \mathrm{~nm} \mathrm{~K}^{-1}$. This represents a more than 20-fold reduction in temperature-sensitivity. Applying Eq. (1) to our experimental results, we derive a filling fraction of $f_{\text {Res } 1}=22.2 \%$ for resonance (1), which is in excellent agreement with the theoretical prediction of $22 \%$ using Eq. (2).

Our stabilization concept is not limited to the $1400 \mathrm{~nm}$ wavelength range. It can easily be extended to other wavelengths, including telecommunication bands. As many polymers exhibit a negative thermo-optic coefficient, it is also feasible to infiltrate a liquid polymer solution into the air holes and subsequently solidify the polymer, e.g., by irradiation with UV light. This would lead to an all-solid temperature-stable $\mathrm{PhC}$ cavity.

The demonstrated temperature-stable optofluidic cavity had a quality factor of $Q \approx 15000$, in the moderate to high range. Such a $Q$-factor would be adequate for applications not requiring exceptionally high spectral resolution. As an example, Chao et al. $^{25}$ were able to demonstrate a biosensor capable of detecting a refractive index change of $10^{-7}$ refractive index units using a resonator with a $Q$ of 20000 . If higher quality factor applications are envisaged, we have demonstrated an optofluidic cavity with a $Q$ of $\approx 60000,{ }^{10}$ and related design studies by others have indicated the possibility of values up to $10^{6} .^{22}$

In conclusion, we have introduced a principle for creating temperature-insensitive cavities in $\mathrm{PhCs}$ based on optofluidics. Thermo-optic stabilization can be achieved by forming a $\mathrm{PhC}$ cavity with suitable dimensions and using a liquid with an appropriate thermo-optic coefficient. Temperatureinsensitive cavities represent a significant advancement toward developing high-sensitivity sensor systems for chemical and biochemical applications, as well as enabling precise nanophotonic devices such as temperature-stable microlasers.

The authors at USYD acknowledge the support of the Australian Research Council through its Federation Fellow, Centre of Excellence and Discovery Grant programs, and the support of the School of Physics, through its Denison Foundation and the International Science Linkages program by the ISL DEST grant. The silicon samples were fabricated in the framework of the EU-FP6 funded ePIXnet Nanostructuring Platform for Photonic Integration (www.nanophotonics.eu). The authors at DTU acknowledge financial support by the Danish Council for Strategic Research (DSF Grant No. 2117-05-0037) and the Danish Council for Technology and Production Sciences (FTP Grant No. 274-07-0379).

${ }^{1}$ K. J. Vahala, Nature (London) 424, 839 (2003).

${ }^{2}$ S. Noda, M. Fujita, and T. Asano, Nat. Photonics 1, 449 (2007).

${ }^{3}$ D. F. Dorfner, T. Hurlimann, T. Zabel, L. H. Frandsen, G. Abstreiter, and J. J. Finley, Appl. Phys. Lett. 93, 181103 (2008).

${ }^{4}$ E. Chow, A. Grot, L. W. Mirkarimi, M. Sigalas, and G. Girolami, Opt. Lett. 29, 1093 (2004).

${ }^{5}$ S. Mandal and D. Erickson, Opt. Express 16, 1623 (2008).

${ }^{6}$ C. Monat, P. Domachuk, and B. J. Eggleton, Nat. Photonics 1, 106 (2007).

${ }^{7}$ D. Erickson, T. Rockwood, T. Emery, A. Scherer, and D. Psaltis, Opt. Lett. 31, 59 (2006).

${ }^{8}$ K. Busch, S. Lölkes, R. B. Wehrspohn, and H. Föll, Photonic CrystalsAdvances in Design, Fabrication, and Characterization (Wiley, Berlin, 2004).

${ }^{9}$ C. L. C. Smith, U. Bog, S. Tomljenovic-Hanic, M. W. Lee, D. K. Wu, L. O'Faolain, C. Monat, C. Grillet, T. F. Krauss, C. Karnutsch, R. C. McPhedran, and B. J. Eggleton, Opt. Express 16, 15887 (2008).

${ }^{10}$ U. Bog, C. L. C. Smith, M. W. Lee, S. Tomljenovic-Hanic, C. Grillet, C. Monat, L. O'Faolain, C. Karnutsch, T. F. Krauss, R. C. McPhedran, and B. J. Eggleton, Opt. Lett. 33, 2206 (2008).

${ }^{11}$ A. Densmore, D. X. Xu, S. Janz, P. Waldron, T. Mischki, G. Lopinski, A. Delâge, J. Lapointe, P. Cheben, B. Lamontagne, and J. H. Schmid, Opt. Lett. 33, 596 (2008).

${ }^{12}$ M. Uenuma and T. Moooka, Opt. Lett. 34, 599 (2009).

${ }^{13}$ T. Wei, Y. Han, Y. Li, H.-L. Tsai, and H. Xiao, Opt. Express 16, 5764 (2008).

${ }^{14}$ S. M. Weiss, M. Molinari, and P. M. Fauchet, Appl. Phys. Lett. 83, 1980 (2003).

${ }^{15}$ N. Mothe, D. Pagnoux, M. C. Phan Huy, V. Dewinter, G. Laffont, and P. Ferdinand, Opt. Express 16, 19018 (2008).

${ }^{16}$ T. Asano, B. S. Song, and S. Noda, Opt. Express 14, 1996 (2006).

${ }^{17}$ F. G. Della Corte, G. Cocorullo, M. Iodice, and I. Rendina, Appl. Phys. Lett. 77, 1614 (2000).

${ }^{18}$ C. B. Kim and C. B. Su, Meas. Sci. Technol. 15, 1683 (2004).

${ }^{19}$ Z. Zhang, P. Zhao, P. Lin, and F. Sun, Polymer 47, 4893 (2006).

${ }^{20}$ N. A. Mortensen, S. Xiao, and J. Pedersen, Microfluid. Nanofluid. 4, 117 (2008).

${ }^{21}$ Cargille Homepage, http://www.cargille.com/.

${ }^{22}$ S. Tomljenovic-Hanic, C. M. de Sterke, and M. J. Steel, Opt. Express 14, 12451 (2006).

${ }^{23}$ H. H. Li, J. Phys. Chem. Ref. Data 9, 561 (1980).

${ }^{24}$ M. W. Lee, C. Grillet, C. G. Poulton, C. Monat, C. L. Smith, E. Mägi, D. Freeman, S. Madden, B. Luther-Davies, and B. J. Eggleton, Opt. Express 16, 13800 (2008).

${ }^{25}$ C.-Y. Chao, W. Fung, and L. J. Guo, IEEE J. Sel. Top. Quantum Electron. 12, 134 (2006). 